\title{
Performance Evaluation of Basketball Referees with an Integration of Ahp and Waspas Methods
}

\author{
Yeter Aytül Dağlı Ekmekçi ${ }^{1}$, Nilsen Kundakc ${ }^{2^{*}}$, Rıdvan Ekmekçi ${ }^{3}$ \\ 1,2,3 Pamukkale University, Department of Sport Management, Denizli, Turkey,
}

\begin{abstract}
.
Basketball referees have to decide quickly and accurately under stress in a limited time and their decisions will affect the results of the match. Therefore, it is important to evaluate the performances of the referees and give them feedback about their performances. In the current performance evaluation system of the basketball referees, criteria weights are considered as equal. In this study, the weights of the performance evaluation criteria were determined with the help of AHP (Analytic Hierarchy Process) method considering the criteria will have different importance degree in the evaluation process. Then, the ranking of the basketball referees according to their performances was obtained by using WASPAS (Weighted Aggregated Sum Product Assessment) method. In this paper, it is aimed to propose a Multi-Criteria Decision Making (MCDM) approach based on the integration of AHP and WASPAS methods to evaluate the performances of basketball referees.
\end{abstract}

Keywords: Performance evaluation, basketball referee, MCDM, AHP, WASPAS

* Corresponding author, e-mail: nilsenk@pau.edu.tr 


\section{Introduction}

Referees have different places and roles for sport. Referees are very hard worker in the field who applies the rules. They have different role such as being a leader, manager, rule maker or mediator. Quick decision making, justice, control the game, good communication, rules apply, and using physical and mental abilities are other important features for referees.

In addition to these, another important feature of the referees is problem solving. While Heppner and Krouskopf (1987) defines problem solving skills as a complex internal and external demands and desires for integration of cognitive and behavioral processes, Bingham (1998), has been described as the process to reach a certain goal to eliminate difficulties that require a series of efforts. Make a quick decision and solve the problem in a right way is a complex procedure under stress. As we know officiating is a stressful job, it is very important to understand their role and give them feedback to have good result.

Evaluation system of the referees in the world is quite similar. There is an observer and evaluator for the organization who gives some digit numbers according to evaluation criteria. But none of them is including weighted criteria process. According to the assumption of this study, there should be priority differences between the evaluation criteria. The first of the actors who can define the weight of the evaluation criteria may be the referees themselves. Thus, awareness can be created about the values and points of view given by the referees according to these evaluation criteria.

In addition, the main purpose of the referees' evaluation process is to direct the referees to manage better competitions. Therefore, it is important for the referees to know the weighted priorities of these evaluation criteria. For these reasons, the referees' selfdevelopment will have a positive impact on the competition and quality of the competition. From this point of view, the opinions of the referees were consulted in determining the priorities of the evaluation criteria in this study and new integrated approach is proposed based on AHP and WASPAS methods for referee performance evaluation.

In the first section of this study, a brief introduction is given. In the second section, AHP method is introduced and the steps of the method is given. In the third section, WASPAS method is explained, literature review is given and also steps of the method is summarized. In the fourth section, application of performance evaluation of basketball referees with the integrated method is given. And finally in the fifth section the results of the application are discussed and recommendations for future studies are given.

\section{AHP Method}

Analytic Hierarchy Process (AHP) was firstly proposed by Saaty in 1980. Later AHP method is widely used as an efficient MCDM method for determining the weights of the criteria and ranking the alternatives. AHP method allows the decision makers to model the 
complex problems in a hierarchical structure that shows the relationship between criteria, sub-criteria and alternatives. This method consists of three main stages; determining the

hierarchical structure, pairwise comparison of alternatives, criteria and finally synthesis of priorities (Dağdeviren et al., 2009).

In the literature, AHP method has been widely used in the studies both in determining the criteria weights and ranking the alternatives. It has been applied in various fields such as selecting the best alternative, planning, optimization, resolving conflict, resource allocations, etc. (Vaidya and Kumar, 2006). A detailed literature review for AHP method has been made by Vaidya \& Kumar (2006) and Russo \& Camanho (2015).

The steps of AHP method can be summarized as:

Step 1. Firstly decision criteria and alternatives of the problem are determined by the decision makers. Later, hierarchical structure of the decision problem is constructed. At the highest level of the hierarchy, the overall goal of this decision problem takes place and the alternatives are at the lowest level. Criteria and sub-criteria are placed between goal and alternatives (Wang et al., 2007).

Step 2. Pairwise comparisons for $n$ criteria are made by the decision makers using Saaty's 1-9 scale shown in Table 1. Based on these pairwise comparisons, matrix $A$ is obtained as given in Eq. 1.

$$
A=\left[a_{i j}\right]=\left[\begin{array}{cccc}
1 & a_{12} & \cdots & a_{1 n} \\
a_{21} & 1 & \cdots & a_{2 n} \\
\vdots & \vdots & \ddots & \vdots \\
a_{n 1} & 1 / a_{2 n} & \cdots & 1
\end{array}\right] ; i, j=1,2, \ldots, n
$$

In this matrix, $a_{i j}>0, a_{j i}=1 / a_{i j}, a_{i i}=1$ and $a_{i j}$ is the decision maker's rating of relative importance of criterion $i$ respect to criterion $j$. If criteria $i$ and $j$ have equal relative importance for the decision maker then $a_{i j}=a_{j i}=1$ (Caputo et al., 2013).

\begin{tabular}{cl}
\multicolumn{2}{c}{ Table 1. Saaty's 1-9 scale } \\
\hline Intensity of importance & ition \\
\hline 1 & importance \\
3 & srate importance \\
5 & g importance \\
7 & strong importance \\
9 & me importance \\
$2,4,6,8$ & nediate values \\
\hline
\end{tabular}

Step 3. From the pairwise comparison matrices, local weights and priorities of elements in the same level are calculated and weight vector is obtained as in Eq. 2.

$$
W=\left[w_{1}, w_{2}, \ldots, w_{n}\right]^{T} \quad i=1,2, \ldots, n
$$


This vector is the normalized principal eigenvector of matrix $A$. The elements of the weight vector are calculated from the normalized pairwise comparison matrix $A$ by taking the average value of the rows as given in Eq. 3 (Caputo et al., 2013),

$$
w_{i}=\frac{1}{n} \sum_{j}\left(\frac{a_{i j}}{\sum_{i} a_{i j}}\right) \quad i, j=1,2, \ldots, n
$$

Step 4. Pairwise comparison matrices of alternatives under each criterion is built by following the procedure in Step 2 . Then, normalized relative rating $b_{i j}$ is computed for each $i$ th alternative respect to any judgment criterion $C_{j}$, in comparison with the other alternatives (Caputo et al, 2013).

Step 5. In this step, firstly the consistency indices $(C l)$ of the pairwise comparison matrices are checked by using the Eq. 4 , then the consistency ratio $(C R)$ is calculated via Eq. 5.

$$
\begin{gathered}
C I=\left(\lambda_{\max }-n\right) /(n-1) \\
C R=C l / R I
\end{gathered}
$$

$\lambda_{\max }$ seen in Eq. 4, is the largest eigenvalue of $A$. In Eq. 5, $R I$ is the average value of $C l$ one would obtain were the entries in $A$ chosen at random, subject that all diagonal entries

\begin{tabular}{|c|c|c|c|c|c|c|c|c|c|c|c|c|}
\hline$n$ & 1 & 2 & 3 & 4 & 5 & 6 & 7 & 8 & 9 & 10 & 11 & 12 \\
\hline $\boldsymbol{R} \boldsymbol{I}$ & 0 & 0 & 0.52 & 0.89 & 1.11 & 1.25 & 1.35 & 1.40 & 1.45 & 1.49 & 1.51 & 1.54 \\
\hline
\end{tabular}
must equal 1 (Caputo et al, 2013). For different $n$ values $R /$ values can be seen on Table 2 .

Table 2. RI values

Source: (Saaty, 2013)

In the case of $C R<0.10$, the decision matrix is said to be consistent. In the case of $C R>$ 0.10 , the comparison matrix is reviewed and necessary changes are made by the decision makers to bring the matrix into a consistent form.

Step 6. In the last step global priorities including global weights and global scores are obtained by aggregating all local priorities with the application of a simple weighted sum. $A$ ranking score $R_{i}$ is calculated for the ith alternative as seen in Equation (6);

$$
R_{i}=\sum_{j} b_{i j} w_{j}
$$

At the end, the final ranking of the alternatives are determined based on these global priorities.

\section{WASPAS Method}

WASPAS (Weighted Aggregated Sum Product Assessment) method was developed firstly by Zavadskas et al. (2012). WASPAS method is an MCDM method that combines the results 
of Weighted Sum Model (WSM) and Weighted Product Model (WPM). According to the joint generalized criterion of weighted aggregation of additive and multiplicative methods, ranking of the alternatives are determined. This method can control the consistency of the alternative rankings by conducting sensitivity analysis within its own process (Chakraborty and Zavadskas, 2014).

WASPAS method has been applied in different fields in the literature. For instance it was used in selection problems to determine and select the best alternative. These includes, selecting the most suitable machining processes (Madić et al., 2014), selecting contractor in the construction industry (Zavadskas et al., 2015A), selecting industrial robot (Karande et al., 2016), selecting personnel (Karabašević et al., 2016; Urosevic et al. 2017), selecting adequate circuit design of lead-zinc froth flotation (Zavadskas et al., 2016A), selecting garage location at the parcel of a single-family residential house (Baušys and Juodagalvienè, 2017), selecting the most suitable server for a department of a textile company (Yurdoğlu and Kundakcl, 2017) and selecting supplier in a PVC carpentry products manufacturing company (Stojic et al., 2018). Another application area of WASPAS method is assessing the alternatives. It was used in assessing building design alternatives (Zavadskas et al., 2013), assessing site alternatives for the construction of a waste incineration plant (Zavadskas et al., 2015B), assessing an indoor environment of apartments in similar brick houses (Zavadskas et al., 2016B). WASPAS method was also proposed for solving manufacturing decision making problems (Chakraborty and Zavadskas, 2014), optimizing non-traditional machining processes (Chakraborty et al., 2015), planning the priority of high tech industries such as nanotechnology industry (Nezhad et al., 2015), evaluating the performances of banks (Akçakanat et al., 2017) and solving solar-wind power station location problem (Nie et al., 2017). In this paper, WASPAS method is used to evaluate the performances of basketball referees. While determining the criteria weights AHP method is used and the ranking of the referees are determined with the help of WASPAS method.

The steps of the WASPAS can be given as (Zavadskas et al. 2012; Chakraborty and Zavadskas, 2014; Chakraborty et al., 2015):

Step 1. Firstly alternatives $A_{\mathrm{i}}(i=1, \ldots, m)$ and criteria $C_{j}(j=1, \ldots, n)$ are determined by the decision makers.

Step 2. Weights of the criteria are determined with the help of an MCDM method such as AHP, SWARA, etc. or decision makers can determine these weights by intuition.

Step 3. After determining the weights of the criteria, decision matrix is constructed as seen in Eq. 7.

$$
X=\left[X_{i j}\right]_{m x n}=\left[\begin{array}{ccc}
X_{11} & \cdots & X_{1 n} \\
\vdots & \ddots & \vdots \\
X_{m 1} & \cdots & X_{m n}
\end{array}\right]
$$

Step 4. Decision matrix is normalized via Eq. 8 or 9 according to the type of the criterion.

For benefit criteria;

$$
\text { wW W. I C B MECONF. Org }
$$




\section{BUSINESS, MANAGEMENT \& ECONOMICS}

$$
r_{i j}=\frac{x_{i j}}{\max _{i} x_{i j}}
$$

For cost criteria;

$$
r_{i j}=\frac{\min _{i} x_{i j}}{x_{i j}}
$$

Step 5. For each alternative total relative importance $Q_{i}^{(1)}$ is calculated based on Weighted Sum Model (WSM) with the help of Eq. 10.

$$
Q_{i}^{(1)}=\sum_{j=1}^{n} w_{j} \cdot r_{i j}
$$

Here; $w_{j}$ indicates the weight of the $\mathrm{j}^{\text {th }}$ criterion.

Step 6. Then, for each alternative total relative importance $Q_{i}^{(2)}$ is calculated based on Weighted Product Model (WPM) by using Eq. 11.

$$
Q_{i}^{(2)}=\prod_{j=1}^{n}\left(r_{i j}\right)^{w_{j}}
$$

Step 7. Finally, total relative importance of $i^{\text {th }}$ alternative is calculated via Eq. 12 . This more generalized equation is developed in order to increase the ranking accuracy and effectiveness of the decision-making process (Chakraborty et al., 2015).

$$
Q_{i}=\lambda Q_{i}^{(1)}+(1-\lambda) Q_{i}^{(2)}
$$

Here; $\lambda$ is coefficient of joint optimality and $\in[0,1]$.

If WSM and WPM has the same importance degree then coefficient of combined optimality is taken as $\lambda=0.5$. When $\lambda=0$ WASPAS method becomes WPM, and when $\lambda=1$ it transformed into WSM (Zavadskas et al., 2012).

Step 8. Alternatives are ranked according to their $Q_{i}$ values. The best alternative would be that one having the highest $Q_{i}$ value.

\section{Application}

In this part, performances of the basketball referees are evaluated with an integrated approach based on AHP and WASPAS methods. In the current performance evaluation system of the basketball referees, criteria weights are considered as equal. Considering the criteria will have different importance degree in the evaluation process, AHP method is used for determining the weights of the criteria. Then, basketball referees are ranked according to their performances with the help of WASPAS method.

In the proposed integrated approach firstly, AHP method is applied in order to determine the weights of the criteria. In the first step, a committee of decision makers is formed from four decision makers $\mathrm{DM}_{1}, \mathrm{DM}_{2}, \mathrm{DM}_{3}, \mathrm{DM}_{4}$. The first decision maker is FIBA instructor, second one is Euroleague referee, third one is Euroleague delegate and referee observer and the last decision maker is high level basketball coach. Then, these decision makers evaluated 10 decision criteria that used in the current evaluation system given in Table 3. 
Table 3. Evaluation criteria

\begin{tabular}{lccc}
\hline & Criteria & \multicolumn{2}{c}{ Criteria } \\
\hline $\mathrm{C}_{1}$ & Competition and self-esteem & $\mathrm{C}_{6}$ & Standard \\
$\mathrm{C}_{2}$ & Physical state & $\mathrm{C}_{7}$ & Teamwork \\
$\mathrm{C}_{3}$ & Foul assessment & $\mathrm{C}_{8}$ & Game control \\
$\mathrm{C}_{4}$ & Violation assessment & $\mathrm{C}_{9}$ & isciplinary implementation \\
$\mathrm{C}_{5}$ & Mechanical & $\mathrm{C}_{10}$ & Overall performance \\
\hline
\end{tabular}

Pairwise comparisons of criteria are made firstly and then pairwise comparisons are made for alternatives under each criterion. The consistency of each comparison matrix is checked and they found as consistent. Later, these four matrices are reduced into one matrix by the help of geometric mean. This obtained matrix is shown in Table 4. Then, this comparison matrix is normalized by using Eq. 3 as given in Table 5.

Table 4. Pairwise comparison matrix

\begin{tabular}{|c|c|c|c|c|c|c|c|c|c|c|c|c|}
\hline \multicolumn{2}{|c|}{$C_{1}$} & \multirow[t]{2}{*}{$C_{2}$} & \multicolumn{2}{|c|}{$C_{3}$} & \multirow{2}{*}{$\frac{z_{4}}{6.00}$} & 25 & 26 & -7 & -8 & \multirow[t]{2}{*}{$\mathrm{C}_{9}$} & \multicolumn{2}{|c|}{$C_{10}$} \\
\hline$C_{1}$ & 1.00 & & 4.28 & 2.51 & & 5.96 & 1.00 & 2.00 & 1.97 & & 4.61 & 5.58 \\
\hline $\mathrm{C}_{2}$ & 0.23 & & 1.00 & 0.71 & 2.11 & 2.45 & 0.24 & 0.43 & 0.64 & & 1.19 & 1.73 \\
\hline$C_{3}$ & 0.40 & & 1.41 & 1.00 & 3.25 & 3.22 & 0.29 & 0.54 & 0.79 & & 1.97 & 2.40 \\
\hline $\mathrm{C}_{4}$ & 0.17 & & 0.47 & 0.31 & 1.00 & 0.84 & 0.16 & 0.21 & 0.34 & & 0.70 & 0.84 \\
\hline $\mathrm{C}_{5}$ & 0.17 & & 0.41 & 0.31 & 1.19 & 1.00 & 0.16 & 0.23 & 0.35 & & 0.74 & 0.93 \\
\hline $\mathrm{C}_{6}$ & 1.00 & & 4.16 & 3.46 & 6.40 & 6.19 & 1.00 & 1.86 & 1.63 & & 4.92 & 5.79 \\
\hline $\mathrm{C}_{7}$ & 0.50 & & 2.34 & 1.86 & 4.74 & 4.36 & 0.54 & 1.00 & 1.09 & & 2.38 & 3.03 \\
\hline $\mathrm{C}_{8}$ & 0.51 & & 1.57 & 1.27 & 2.91 & 2.83 & 0.61 & 0.91 & 1.00 & & 2.99 & 3.94 \\
\hline $\mathrm{C}_{9}$ & 0.22 & & 0.84 & 0.51 & 1.43 & 1.35 & 0.20 & 0.42 & 0.33 & & 1.00 & 1.68 \\
\hline$C_{10}$ & 0.18 & & 0.58 & 0.42 & 1.19 & 1.07 & 0.17 & 0.33 & 0.25 & & 0.59 & 1.00 \\
\hline
\end{tabular}

Table 5. Normalized matrix

\begin{tabular}{rrrrrrrrrrr}
\hline & $\mathrm{C}_{1}$ & $\mathrm{C}_{2}$ & $\mathrm{C}_{3}$ & $\mathrm{C}_{4}$ & $\mathrm{C}_{5}$ & $\mathrm{C}_{6}$ & $\mathrm{C}_{7}$ & $\mathrm{C}_{8}$ & $\mathrm{C}_{9}$ & $\mathrm{C}_{10}$ \\
\hline $\mathrm{C}_{1}$ & 0.23 & 0.25 & 0.20 & 0.20 & 0.20 & 0.23 & 0.25 & 0.23 & 0.22 & 0.21 \\
$\mathrm{C}_{\mathbf{2}}$ & 0.05 & 0.06 & 0.06 & 0.07 & 0.08 & 0.05 & 0.05 & 0.08 & 0.06 & 0.06 \\
$\mathrm{C}_{3}$ & 0.09 & 0.08 & 0.08 & 0.11 & 0.11 & 0.07 & 0.07 & 0.09 & 0.09 & 0.09 \\
$\mathrm{C}_{4}$ & 0.04 & 0.03 & 0.02 & 0.03 & 0.03 & 0.04 & 0.03 & 0.04 & 0.03 & 0.03 \\
$\mathrm{C}_{5}$ & 0.04 & 0.02 & 0.03 & 0.04 & 0.03 & 0.04 & 0.03 & 0.04 & 0.04 & 0.03 \\
$\mathrm{C}_{6}$ & 0.23 & 0.24 & 0.28 & 0.21 & 0.21 & 0.23 & 0.23 & 0.19 & 0.23 & 0.21 \\
$\mathrm{C}_{7}$ & 0.11 & 0.14 & 0.15 & 0.16 & 0.15 & 0.12 & 0.13 & 0.13 & 0.11 & 0.11 \\
$\mathrm{C}_{\mathbf{8}}$ & 0.12 & 0.09 & 0.10 & 0.10 & 0.10 & 0.14 & 0.12 & 0.12 & 0.14 & 0.15 \\
$\mathrm{C}_{9}$ & 0.05 & 0.05 & 0.04 & 0.05 & 0.05 & 0.05 & 0.05 & 0.04 & 0.05 & 0.06 \\
$\mathrm{C}_{\mathbf{1 0}}$ & 0.04 & 0.03 & 0.03 & 0.04 & 0.04 & 0.04 & 0.04 & 0.03 & 0.03 & 0.04 \\
\hline
\end{tabular}

Finally, the weights of the criteria are calculated by taking the averages of the each row of the normalized matrix. The obtained weights for each criterion is given in Table 6 . 
Table 6. Weights of the criteria

\begin{tabular}{lrr}
\hline & Criteria & Weights \\
\hline $\mathbf{C}_{6}$ & Standard & 0.23 \\
$\mathbf{C}_{1}$ & Competition and self-esteem & 0.22 \\
$\mathbf{C}_{\mathbf{7}}$ & Teamwork & 0.13 \\
$\mathbf{C}_{\mathbf{8}}$ & Game control & 0.12 \\
$\mathbf{C}_{\mathbf{3}}$ & Foul assessment & 0.09 \\
$\mathbf{C}_{\mathbf{2}}$ & Physical state & 0.06 \\
$\mathbf{C}_{9}$ & Disciplinary implementation & 0.05 \\
$\mathbf{C}_{10}$ & Overall performance & 0.04 \\
$\mathbf{C}_{5}$ & Mechanical & 0.03 \\
$\mathbf{C}_{4}$ & Violation assessment & 0,03 \\
\hline
\end{tabular}

After determining the criteria weights with AHP method, steps of WASPAS method are followed in order to determine the ranking of the basketball referees. Firstly, decision matrix is formed. This matrix includes the performance values of basketball referees.

Table 7. Decision Matrix

\begin{tabular}{|c|c|c|c|c|c|c|c|c|c|c|}
\hline Referees & $\mathrm{C}_{1}$ & $C_{2}$ & $C_{3}$ & $\mathrm{C}_{4}$ & $C_{5}$ & $C_{6}$ & $C_{7}$ & $\mathrm{C}_{8}$ & $\mathrm{C}_{9}$ & $\mathrm{C}_{10}$ \\
\hline $\mathbf{R}_{\mathbf{1}}$ & 8.88 & 8.75 & 7.75 & 8.00 & 7.88 & 8.13 & 8.13 & 7.88 & 8.25 & 8.25 \\
\hline $\mathbf{R}_{\mathbf{2}}$ & 8.50 & 7.75 & 7.13 & 7.63 & 6.75 & 7.63 & 7.13 & 7.63 & 7.63 & 7.38 \\
\hline $\mathbf{R}_{\mathbf{3}}$ & 8.60 & 8.40 & 7.00 & 7.80 & 7.00 & 7.20 & 7.60 & 7.60 & 7.80 & 7.40 \\
\hline $\mathbf{R}_{\mathbf{4}}$ & 9.00 & 8.25 & 7.50 & 8.13 & 8.00 & 7.88 & 8.38 & 7.88 & 8.13 & 7.75 \\
\hline $\mathbf{R}_{\mathbf{5}}$ & 8.75 & 8.25 & 6.75 & 7.25 & 7.25 & 7.00 & 7.00 & 7.75 & 7.25 & 7.25 \\
\hline $\mathbf{R}_{6}$ & 8.67 & 8.67 & 7.00 & 7.33 & 7.67 & 7.33 & 7.67 & 6.83 & 7.00 & 7.00 \\
\hline $\mathbf{R}_{7}$ & 9.29 & 9.14 & 7.86 & 7.86 & 7.86 & 8.14 & 8.43 & 8.14 & 8.29 & 8.14 \\
\hline $\mathbf{R}_{\mathbf{8}}$ & 8.67 & 7.83 & 6.83 & 7.00 & 7.00 & 7.17 & 7.00 & 6.83 & 7.00 & 7.00 \\
\hline $\mathbf{R}_{\mathbf{9}}$ & 8.63 & 8.75 & 6.88 & 7.00 & 7.00 & 6.88 & 7.13 & 7.38 & 7.00 & 7.13 \\
\hline $\mathbf{R}_{10}$ & 8.75 & 8.25 & 7.13 & 7.25 & 7.25 & 6.88 & 7.13 & 7.13 & 7.13 & 7.00 \\
\hline $\mathbf{R}_{11}$ & 8.71 & 8.71 & 6.71 & 7.43 & 7.43 & 7.29 & 7.57 & 7.43 & 7.43 & 7.43 \\
\hline $\mathbf{R}_{12}$ & 9.00 & 8.50 & 7.50 & 7.63 & 7.63 & 7.50 & 8.13 & 7.75 & 7.88 & 7.88 \\
\hline $\mathbf{R}_{13}$ & 9.00 & 7.57 & 8.00 & 7.71 & 8.00 & 7.57 & 7.71 & 7.57 & 7.57 & 7.86 \\
\hline $\mathbf{R}_{14}$ & 9.29 & 8.71 & 7.71 & 8.14 & 8.00 & 7.71 & 8.29 & 8.00 & 8.29 & 8.00 \\
\hline $\mathbf{R}_{15}$ & 8.50 & 8.25 & 7.25 & 7.25 & 7.25 & 7.50 & 7.75 & 7.25 & 7.50 & 7.25 \\
\hline $\mathbf{R}_{16}$ & 8.88 & 9.00 & 7.25 & 7.50 & 7.75 & 7.25 & 7.63 & 7.63 & 7.38 & 7.63 \\
\hline $\mathbf{R}_{17}$ & 9.67 & 9.33 & 7.67 & 8.33 & 8.00 & 8.33 & 8.00 & 8.00 & 8.17 & 8.00 \\
\hline $\mathbf{R}_{18}$ & 9.25 & 9.13 & 7.50 & 7.88 & 7.88 & 7.50 & 7.88 & 7.63 & 7.88 & 7.88 \\
\hline $\mathbf{R}_{19}$ & 9.00 & 9.44 & 7.89 & 8.22 & 7.78 & 7.78 & 7.89 & 8.33 & 8.33 & 8.00 \\
\hline $\mathbf{R}_{\mathbf{2 0}}$ & 8.88 & 6.63 & 7.63 & 7.50 & 7.25 & 7.88 & 8.25 & 8.13 & 8.25 & 7.88 \\
\hline $\mathbf{R}_{\mathbf{2 1}}$ & 8.50 & 8.25 & 7.00 & 7.25 & 7.50 & 7.50 & 7.75 & 7.50 & 7.25 & 7.25 \\
\hline $\mathbf{R}_{22}$ & 8.71 & 8.29 & 8.14 & 7.86 & 8.29 & 8.14 & 8.29 & 8.57 & 8.57 & 8.29 \\
\hline $\mathbf{R}_{\mathbf{2 3}}$ & 9.00 & 8.20 & 7.40 & 7.40 & 7.60 & 7.60 & 7.80 & 7.60 & 7.40 & 7.60 \\
\hline $\mathbf{R}_{24}$ & 9.00 & 8.83 & 7.33 & 7.83 & 7.50 & 7.17 & 7.50 & 7.67 & 7.83 & 7.50 \\
\hline $\mathbf{R}_{25}$ & 8.88 & 7.88 & 7.38 & 7.63 & 7.50 & 7.38 & 7.88 & 7.63 & 8.00 & 7.63 \\
\hline $\mathbf{R}_{26}$ & 8.80 & 8.20 & 7.40 & 7.20 & 7.60 & 7.60 & 7.60 & 7.20 & 7.80 & 7.60 \\
\hline $\mathbf{R}_{\mathbf{2 7}}$ & 8.83 & 8.33 & 7.00 & 7.67 & 7.67 & 7.33 & 8.33 & 7.50 & 7.67 & 7.50 \\
\hline $\mathbf{R}_{\mathbf{2 8}}$ & 9.13 & 8.88 & 8.13 & 7.88 & 8.38 & 8.13 & 8.25 & 8.00 & 8.13 & 8.13 \\
\hline
\end{tabular}




\begin{tabular}{llllllllllll}
$\mathbf{R}_{\mathbf{2 9}}$ & 9.00 & 3.50 & 5.50 & .50 & .25 & .25 & 7.25 & 5.75 & 7.00 & 7.00 \\
$\mathbf{R}_{\mathbf{3 0}}$ & 8.50 & 7.50 & 7.33 & .17 & .17 & .17 & 7.50 & 7.50 & 8.00 & 7.50 & \\
\hline
\end{tabular}

Decision matrix is normalized by using Eq. 8 as all the criteria are benefit criteria. Then normalized decision matrix is formed as seen in Table 8.

Table 8. Normalized Decision Matrix

\begin{tabular}{|c|c|c|c|c|c|c|c|c|c|c|}
\hline Referees & $\mathrm{C}_{1}$ & $\mathrm{C}_{2}$ & $C_{3}$ & $\mathrm{C}_{4}$ & $C_{5}$ & $\mathrm{C}_{6}$ & $\mathrm{C}_{7}$ & $\mathrm{C}_{8}$ & $\mathrm{C}_{9}$ & $C_{10}$ \\
\hline $\mathbf{R}_{1}$ & 0.918 & 0.926 & 0.952 & 0.960 & 0.940 & 0.975 & 0.964 & 0.919 & 0.963 & 0.996 \\
\hline $\mathbf{R}_{\mathbf{2}}$ & 0.879 & 0.821 & 0.875 & 0.915 & 0.806 & 0.915 & 0.845 & 0.890 & 0.890 & 0.890 \\
\hline $\mathbf{R}_{\mathbf{3}}$ & 0.890 & 0.889 & 0.860 & 0.936 & 0.836 & 0.864 & 0.902 & 0.887 & 0.910 & 0.893 \\
\hline $\mathbf{R}_{\mathbf{4}}$ & 0.931 & 0.874 & 0.921 & 0.975 & 0.955 & 0.945 & 0.994 & 0.919 & 0.948 & 0.935 \\
\hline $\mathbf{R}_{5}$ & 0.905 & 0.874 & 0.829 & 0.870 & 0.866 & 0.840 & 0.831 & 0.904 & 0.846 & 0.875 \\
\hline $\mathbf{R}_{6}$ & 0.897 & 0.918 & 0.860 & 0.880 & 0.915 & 0.880 & 0.910 & 0.797 & 0.817 & 0.845 \\
\hline $\mathbf{R}_{7}$ & 0.961 & 0.968 & 0.965 & 0.943 & 0.938 & 0.977 & 1.000 & 0.950 & 0.967 & 0.983 \\
\hline $\mathbf{R}_{\mathbf{8}}$ & 0.897 & 0.829 & 0.839 & 0.840 & 0.836 & 0.860 & 0.831 & 0.797 & 0.817 & 0.845 \\
\hline $\mathbf{R}_{\mathbf{9}}$ & 0.892 & 0.926 & 0.844 & 0.840 & 0.836 & 0.825 & 0.845 & 0.860 & 0.817 & 0.860 \\
\hline $\mathbf{R}_{10}$ & 0.905 & 0.874 & 0.875 & 0.870 & 0.866 & 0.825 & 0.845 & 0.831 & 0.831 & 0.845 \\
\hline $\mathbf{R}_{11}$ & 0.901 & 0.923 & 0.825 & 0.891 & 0.887 & 0.874 & 0.898 & 0.867 & 0.867 & 0.897 \\
\hline $\mathbf{R}_{12}$ & 0.931 & 0.900 & 0.921 & 0.915 & 0.910 & 0.900 & 0.964 & 0.904 & 0.919 & 0.950 \\
\hline $\mathbf{R}_{13}$ & 0.931 & 0.802 & 0.982 & 0.926 & 0.955 & 0.909 & 0.915 & 0.883 & 0.883 & 0.948 \\
\hline $\mathbf{R}_{14}$ & 0.961 & 0.923 & 0.947 & 0.977 & 0.955 & 0.926 & 0.983 & 0.933 & 0.967 & 0.966 \\
\hline $\mathbf{R}_{15}$ & 0.879 & 0.874 & 0.890 & 0.870 & 0.866 & 0.900 & 0.919 & 0.846 & 0.875 & 0.875 \\
\hline $\mathbf{R}_{16}$ & 0.918 & 0.953 & 0.890 & 0.900 & 0.925 & 0.870 & 0.905 & 0.890 & 0.860 & 0.920 \\
\hline $\mathbf{R}_{17}$ & 1.000 & 0.988 & 0.942 & 1.000 & 0.955 & 1.000 & 0.949 & 0.933 & 0.953 & 0.966 \\
\hline $\mathbf{R}_{18}$ & 0.957 & 0.966 & 0.921 & 0.945 & 0.940 & 0.900 & 0.934 & 0.890 & 0.919 & 0.950 \\
\hline $\mathbf{R}_{19}$ & 0.931 & 1.000 & 0.969 & 0.987 & 0.929 & 0.933 & 0.936 & 0.972 & 0.972 & 0.966 \\
\hline $\mathbf{R}_{20}$ & 0.918 & 0.701 & 0.936 & 0.900 & 0.866 & 0.945 & 0.979 & 0.948 & 0.963 & 0.950 \\
\hline $\mathbf{R}_{21}$ & 0.879 & 0.874 & 0.860 & 0.870 & 0.896 & 0.900 & 0.919 & 0.875 & 0.846 & 0.875 \\
\hline $\mathbf{R}_{22}$ & 0.901 & 0.877 & 1.000 & 0.943 & 0.989 & 0.977 & 0.983 & 1.000 & 1.000 & 1.000 \\
\hline $\mathbf{R}_{23}$ & 0.931 & 0.868 & 0.909 & 0.888 & 0.907 & 0.912 & 0.925 & 0.887 & 0.863 & 0.917 \\
\hline $\mathbf{R}_{\mathbf{2 4}}$ & 0.931 & 0.935 & 0.901 & 0.940 & 0.896 & 0.860 & 0.890 & 0.894 & 0.914 & 0.905 \\
\hline $\mathbf{R}_{25}$ & 0.918 & 0.834 & 0.906 & 0.915 & 0.896 & 0.885 & 0.934 & 0.890 & 0.933 & 0.920 \\
\hline $\mathbf{R}_{\mathbf{2 6}}$ & 0.910 & 0.868 & 0.909 & 0.864 & 0.907 & 0.912 & 0.902 & 0.840 & 0.910 & 0.917 \\
\hline $\mathbf{R}_{\mathbf{2 7}}$ & 0.914 & 0.882 & 0.860 & 0.920 & 0.915 & 0.880 & 0.989 & 0.875 & 0.894 & 0.905 \\
\hline $\mathbf{R}_{\mathbf{2 8}}$ & 0.944 & 0.940 & 0.998 & 0.945 & 1.000 & 0.975 & 0.979 & 0.933 & 0.948 & 0.981 \\
\hline $\mathbf{R}_{\mathbf{2 9}}$ & 0.931 & 0.900 & 0.798 & 0.900 & 0.866 & 0.750 & 0.860 & 0.788 & 0.817 & 0.845 \\
\hline $\mathbf{R}_{\mathbf{3 0}}$ & 0.879 & 0.794 & 0.901 & 0.860 & 0.856 & 0.860 & 0.890 & 0.875 & 0.933 & 0.905 \\
\hline
\end{tabular}

Total relative importance $Q_{i}^{(1)}$ is calculated with the help of Eq. 10 for all referees. In this equation weight values obtained with AHP method are considered. These total relative importance values for the referees are given on Table 9. 
Table 9. Weighed normalized decision matrix and total relative importance $Q_{i}^{(1)}$

\begin{tabular}{|c|c|c|c|c|c|c|c|c|c|c|c|}
\hline Referees & $C_{1}$ & $C_{2}$ & $C_{3}$ & $\mathrm{C}_{4}$ & $C_{5}$ & $\mathrm{C}_{6}$ & $C_{7}$ & $C_{8}$ & $C_{9}$ & $C_{10}$ & $Q_{i}^{(1)}$ \\
\hline $\mathbf{R}_{\mathbf{1}}$ & 0.202 & 0.056 & 0.086 & 0.029 & 0.028 & 0.224 & 0.125 & 0.110 & 0.048 & 0.040 & 0.948 \\
\hline $\mathbf{R}_{\mathbf{2}}$ & 0.193 & 0.049 & 0.079 & 0.027 & 0.024 & 0.210 & 0.110 & 0.107 & 0.044 & 0.036 & 0.880 \\
\hline $\mathbf{R}_{\mathbf{3}}$ & 0.196 & 0.053 & 0.077 & 0.028 & 0.025 & 0.199 & 0.117 & 0.106 & 0.046 & 0.036 & 0.883 \\
\hline $\mathbf{R}_{4}$ & 0.205 & 0.052 & 0.083 & 0.029 & 0.029 & 0.217 & 0.129 & 0.110 & 0.047 & 0.037 & 0.940 \\
\hline $\mathbf{R}_{5}$ & 0.199 & 0.052 & 0.075 & 0.026 & 0.026 & 0.193 & 0.108 & 0.109 & 0.042 & 0.035 & 0.865 \\
\hline $\mathbf{R}_{6}$ & 0.197 & 0.055 & 0.077 & 0.026 & 0.027 & 0.202 & 0.118 & 0.096 & 0.041 & 0.034 & 0.874 \\
\hline $\mathbf{R}_{7}$ & 0.211 & 0.058 & 0.087 & 0.028 & 0.028 & 0.225 & 0.130 & 0.114 & 0.048 & 0.039 & 0.969 \\
\hline $\mathbf{R}_{8}$ & 0.197 & 0.050 & 0.076 & 0.025 & 0.025 & 0.198 & 0.108 & 0.096 & 0.041 & 0.034 & 0.849 \\
\hline $\mathbf{R}_{\mathbf{g}}$ & 0.196 & 0.056 & 0.076 & 0.025 & 0.025 & 0.190 & 0.110 & 0.103 & 0.041 & 0.034 & 0.856 \\
\hline $\mathbf{R}_{10}$ & 0.199 & 0.052 & 0.079 & 0.026 & 0.026 & 0.190 & 0.110 & 0.100 & 0.042 & 0.034 & 0.857 \\
\hline $\mathbf{R}_{11}$ & 0.198 & 0.055 & 0.074 & 0.027 & 0.027 & 0.201 & 0.117 & 0.104 & 0.043 & 0.036 & 0.882 \\
\hline $\mathbf{R}_{12}$ & 0.205 & 0.054 & 0.083 & 0.027 & 0.027 & 0.207 & 0.125 & 0.109 & 0.046 & 0.038 & 0.921 \\
\hline $\mathbf{R}_{13}$ & 0.205 & 0.048 & 0.088 & 0.028 & 0.029 & 0.209 & 0.119 & 0.106 & 0.044 & 0.038 & 0.914 \\
\hline $\mathbf{R}_{14}$ & 0.211 & 0.055 & 0.085 & 0.029 & 0.029 & 0.213 & 0.128 & 0.112 & 0.048 & 0.039 & 0.950 \\
\hline $\mathbf{R}_{15}$ & 0.193 & 0.052 & 0.080 & 0.026 & 0.026 & 0.207 & 0.120 & 0.102 & 0.044 & 0.035 & 0.885 \\
\hline $\mathbf{R}_{16}$ & 0.202 & 0.057 & 0.080 & 0.027 & 0.028 & 0.200 & 0.118 & 0.107 & 0.043 & 0.037 & 0.898 \\
\hline $\mathbf{R}_{17}$ & 0.220 & 0.059 & 0.085 & 0.030 & 0.029 & 0.230 & 0.123 & 0.112 & 0.048 & 0.039 & 0.974 \\
\hline $\mathbf{R}_{18}$ & 0.211 & 0.058 & 0.083 & 0.028 & 0.028 & 0.207 & 0.121 & 0.107 & 0.046 & 0.038 & 0.927 \\
\hline $\mathbf{R}_{19}$ & 0.205 & 0.060 & 0.087 & 0.030 & 0.028 & 0.215 & 0.122 & 0.117 & 0.049 & 0.039 & 0.950 \\
\hline $\mathbf{R}_{20}$ & 0.202 & 0.042 & 0.084 & 0.027 & 0.026 & 0.217 & 0.127 & 0.114 & 0.048 & 0.038 & 0.926 \\
\hline $\mathbf{R}_{21}$ & 0.193 & 0.052 & 0.077 & 0.026 & 0.027 & 0.207 & 0.120 & 0.105 & 0.042 & 0.035 & 0.885 \\
\hline $\mathbf{R}_{22}$ & 0.198 & 0.053 & 0.090 & 0.028 & 0.030 & 0.225 & 0.128 & 0.120 & 0.050 & 0.040 & 0.961 \\
\hline $\mathbf{R}_{23}$ & 0.205 & 0.052 & 0.082 & 0.027 & 0.027 & 0.210 & 0.120 & 0.106 & 0.043 & 0.037 & 0.909 \\
\hline $\mathbf{R}_{\mathbf{2 4}}$ & 0.205 & 0.056 & 0.081 & 0.028 & 0.027 & 0.198 & 0.116 & 0.107 & 0.046 & 0.036 & 0.900 \\
\hline $\mathbf{R}_{25}$ & 0.202 & 0.050 & 0.082 & 0.027 & 0.027 & 0.204 & 0.121 & 0.107 & 0.047 & 0.037 & 0.903 \\
\hline $\mathbf{R}_{26}$ & 0.200 & 0.052 & 0.082 & 0.026 & 0.027 & 0.210 & 0.117 & 0.101 & 0.046 & 0.037 & 0.897 \\
\hline $\mathbf{R}_{27}$ & 0.201 & 0.053 & 0.077 & 0.028 & 0.027 & 0.202 & 0.129 & 0.105 & 0.045 & 0.036 & 0.903 \\
\hline $\mathbf{R}_{28}$ & 0.208 & 0.056 & 0.090 & 0.028 & 0.030 & 0.224 & 0.127 & 0.112 & 0.047 & 0.039 & 0.962 \\
\hline $\mathbf{R}_{29}$ & 0.205 & 0.054 & 0.072 & 0.027 & 0.026 & 0.173 & 0.112 & 0.095 & 0.041 & 0.034 & 0.837 \\
\hline $\mathbf{R}_{\mathbf{3 0}}$ & 0.193 & 0.048 & 0.081 & 0.026 & 0.026 & 0.198 & 0.116 & 0.105 & 0.047 & 0.036 & 0.875 \\
\hline
\end{tabular}

Later, total relative importance $Q_{i}^{(2)}$ for each alternative are calculated by using Eq. 11 and given in Table 10. 
Table 10. Weighed normalized decision matrix and total relative importance $Q_{i}^{(2)}$

\begin{tabular}{|c|c|c|c|c|c|c|c|c|c|c|c|}
\hline Referees & $C_{1}$ & $C_{2}$ & $C_{3}$ & $\mathrm{C}_{4}$ & $C_{5}$ & $C_{6}$ & $C_{7}$ & $C_{8}$ & $C_{9}$ & $C_{10}$ & $Q_{i}^{(2)}$ \\
\hline $\mathbf{R}_{\mathbf{1}}$ & .981 & 0.995 & 0.996 & 0.999 & 0.998 & 0.994 & 0.995 & 0.990 & 0.998 & 1.000 & 0.948 \\
\hline $\mathbf{R}_{2}$ & .972 & 0.988 & .988 & 0.997 & 0.994 & 0.980 & 0.978 & 0.986 & & 0.995 & 0.880 \\
\hline $\mathbf{R}_{\mathbf{3}}$ & 0.975 & 0.993 & 0.986 & 0.998 & 0.995 & 0.967 & 0.987 & & 0.995 & 0.995 & 0.883 \\
\hline $\mathbf{R}_{\mathbf{4}}$ & 0.984 & 0.992 & 0.993 & 0.999 & 0.999 & 0.987 & 0.999 & 0.990 & 0.997 & 0.997 & 0.939 \\
\hline $\mathbf{R}_{5}$ & 0.978 & 0.992 & 0.983 & 0.996 & 0.996 & 0.961 & 0.976 & 0.988 & 0.992 & 0.995 & 0.865 \\
\hline $\mathbf{R}_{6}$ & 0.976 & 0.995 & 0.986 & 0.996 & 0.997 & 0.971 & 0.988 & 0.973 & 0.990 & 0.993 & 0.874 \\
\hline $\mathbf{R}_{\mathbf{7}}$ & .991 & 0.998 & 0.997 & 0.998 & 0.998 & 0.995 & 1.000 & & & & 0.969 \\
\hline $\mathbf{R}_{8}$ & 0.976 & 0.989 & 0.984 & 0.995 & 0.995 & 0.9 & 0.976 & 0.973 & & 0.993 & 0.848 \\
\hline $\mathbf{R g}_{\mathbf{g}}$ & 0.975 & 0.995 & 0.985 & 0.995 & 0.995 & 0.9 & 0.978 & 0.982 & 90 & 994 & 0.856 \\
\hline $\mathbf{R}_{10}$ & 0.978 & 0.992 & 0.988 & 0.996 & 0.996 & 0.9 & 0.978 & 0.978 & & 93 & 0.857 \\
\hline $\mathbf{R}_{11}$ & 0.977 & 0.995 & 0.983 & 0.997 & 0.996 & 0.970 & 0.986 & 0.983 & 0.993 & 0.996 & 0.882 \\
\hline $\mathbf{R}_{12}$ & 34 & 0.994 & 0.9 & 0.99 & 0.9 & 0.9 & & & & & 0.921 \\
\hline $\mathbf{R}_{13}$ & 0.984 & 0.987 & 0.998 & 0.998 & 0.999 & 0.978 & 0.989 & 0.985 & 0.9 & 0.998 & 0.913 \\
\hline $\mathbf{R}_{14}$ & 0.991 & 0.995 & 0.995 & 0.999 & 0.999 & 0.9 & 0.998 & 0.9 & & 0.999 & 0.949 \\
\hline $\mathbf{R}_{15}$ & & & & & & & & & & & 0.885 \\
\hline $\mathbf{R}_{16}$ & 0.981 & 0.997 & 0.990 & 0.997 & 0.998 & 0.968 & 0.987 & 0.986 & 0.993 & 0.997 & 0.898 \\
\hline $\mathbf{R}_{17}$ & 1.000 & 0.999 & 0.995 & 1.000 & 0.999 & 1.000 & 0.993 & 0.992 & 0.998 & 0.999 & 0.974 \\
\hline $\mathbf{R}_{18}$ & 0.990 & 0.998 & 0.99 & 0.998 & 0.998 & 0.9 & 0.991 & 0.986 & 0.9 & 0.998 & 0.927 \\
\hline $\mathbf{R}_{19}$ & 0.984 & 1.000 & 0.997 & 1.000 & 0.998 & 0.984 & 0.991 & 0.997 & 0.999 & 0.999 & 0.949 \\
\hline $\mathbf{R}_{\mathbf{2 0}}$ & & & & & & & & & & & 0.923 \\
\hline $\mathbf{R}_{21}$ & 0.972 & 0.992 & 0.986 & 0.996 & 0.997 & 0.9 & 0.989 & 0.984 & 0.992 & 0.995 & 0.885 \\
\hline $\mathbf{R}_{\mathbf{2 2}}$ & 0.977 & 0.992 & 1.000 & 0.998 & 1.000 & 0.995 & 0.998 & 1.000 & 1.000 & 1.000 & 0.961 \\
\hline $\mathbf{R}_{\mathbf{2 3}}$ & 0.984 & 0.992 & 0.991 & 0.996 & 0.997 & 0.979 & 0.990 & & & 0.9 & 0.909 \\
\hline $\mathbf{R}_{24}$ & 0.984 & 0.996 & 0.991 & 0.998 & 0.997 & 0.966 & 0.985 & 0.987 & 0.996 & 0.996 & 0.899 \\
\hline $\mathbf{R}_{\mathbf{2 5}}$ & & 0.989 & 0.991 & 0.997 & 0.997 & 0.972 & & & & 0.997 & 0.903 \\
\hline $\mathbf{R}_{26}$ & 0.980 & 0.992 & 0.991 & 0.996 & 0.997 & 0.979 & 0.987 & 0.979 & 0.995 & 0.997 & 0.897 \\
\hline $\mathbf{R}_{27}$ & 0.980 & 0.993 & 0.986 & 0.998 & 0.997 & 0.971 & 0.999 & 0.984 & 0.994 & 0.996 & 0.903 \\
\hline $\mathbf{R}_{\mathbf{2 8}}$ & 0.987 & 0.996 & 1.000 & 0.998 & 1.000 & 0.994 & 0.997 & 0.992 & 0.997 & 0.999 & 0.962 \\
\hline $\mathbf{R}_{29}$ & 0.984 & 0.994 & 0.980 & 0.997 & 0.996 & 0.936 & 0.981 & 0.972 & 0.990 & 0.993 & 0.834 \\
\hline $\mathbf{R}_{\mathbf{3 0}}$ & 0.972 & 0.986 & 0.991 & 0.995 & 0.995 & 0.966 & 0.985 & 0.984 & 0.997 & 0.996 & 0.875 \\
\hline
\end{tabular}

Finally, total relative importance of the referees are calculated with the help of Eq. 12. Here coefficient of combined optimality is taken as $\lambda=0.5$. Total relative importance values of referees are given on Table 11. 


\section{$2^{\text {nd }}$ International Conference on BUSINESS, MANAGEMENT \& ECONOMICS}

Table 11. Total relative importance of the referees

\begin{tabular}{cccccc}
\hline Zeferees & $\boldsymbol{Q}_{\boldsymbol{i}}$ & 2eferees & $\boldsymbol{Q}_{\boldsymbol{i}}$ & 2eferees & $\boldsymbol{Q}_{\boldsymbol{i}}$ \\
\hline $\mathbf{R}_{\mathbf{1}}$ & .948 & $\mathbf{R}_{\mathbf{1 1}}$ & .882 & $\mathbf{R}_{\mathbf{2 1}}$ & .885 \\
$\mathbf{R}_{\mathbf{2}}$ & .880 & $\mathbf{R}_{\mathbf{1 2}}$ & .921 & $\mathbf{R}_{\mathbf{2 2}}$ & .961 \\
$\mathbf{R}_{\mathbf{3}}$ & .883 & $\mathbf{R}_{\mathbf{1 3}}$ & .913 & $\mathbf{R}_{\mathbf{2 3}}$ & .909 \\
$\mathbf{R}_{\mathbf{4}}$ & .939 & $\mathbf{R}_{\mathbf{1 4}}$ & .949 & $\mathbf{R}_{\mathbf{2 4}}$ & .900 \\
$\mathbf{R}_{\mathbf{5}}$ & .865 & $\mathbf{R}_{\mathbf{1 5}}$ & .885 & $\mathbf{R}_{\mathbf{2 5}}$ & .903 \\
$\mathbf{R}_{\mathbf{6}}$ & .874 & $\mathbf{R}_{\mathbf{1 6}}$ & .898 & $\mathbf{R}_{\mathbf{2 6}}$ & .897 \\
$\mathbf{R}_{\mathbf{7}}$ & .969 & $\mathbf{R}_{\mathbf{1 7}}$ & .974 & $\mathbf{R}_{\mathbf{2 7}}$ & .903 \\
$\mathbf{R}_{\mathbf{8}}$ & .849 & $\mathbf{R}_{\mathbf{1 8}}$ & .927 & $\mathbf{R}_{\mathbf{2 8}}$ & .962 \\
$\mathbf{R}_{\mathbf{9}}$ & .856 & $\mathbf{R}_{\mathbf{1 9}}$ & .950 & $\mathbf{R}_{\mathbf{2 9}}$ & .836 \\
$\mathbf{R}_{\mathbf{1 0}}$ & .857 & $\mathbf{R}_{\mathbf{2 0}}$ & .925 & $\mathbf{R}_{\mathbf{3 0}}$ & .875 \\
\hline
\end{tabular}

At the end, basketball referees are ranked according to their $Q_{i}$ values in descending order. Their rankings are given on Table 12.

Table 12. Ranking results of basketball referees

\begin{tabular}{cccccc}
\hline eferees & $\boldsymbol{Q}_{\boldsymbol{i}}$ & eferees & $\boldsymbol{Q}_{\boldsymbol{i}}$ & eferees & $\boldsymbol{Q}_{\boldsymbol{i}}$ \\
\hline $\mathbf{R}_{\mathbf{1 7}}$ & 0.974 & $\mathbf{R}_{\mathbf{1 2}}$ & 0.921 & $\mathbf{R}_{\mathbf{3}}$ & 0.883 \\
$\mathbf{R}_{\mathbf{7}}$ & 0.969 & $\mathbf{R}_{\mathbf{1 3}}$ & 0.913 & $\mathbf{R}_{\mathbf{1 1}}$ & 0.882 \\
$\mathbf{R}_{\mathbf{2 8}}$ & 0.962 & $\mathbf{R}_{\mathbf{2 3}}$ & 0.909 & $\mathbf{R}_{\mathbf{2}}$ & 0.880 \\
$\mathbf{R}_{\mathbf{2 2}}$ & 0.961 & $\mathbf{R}_{\mathbf{2 5}}$ & 0.903 & $\mathbf{R}_{\mathbf{3 0}}$ & 0.875 \\
$\mathbf{R}_{\mathbf{1 9}}$ & 0.950 & $\mathbf{R}_{\mathbf{2 7}}$ & 0.903 & $\mathbf{R}_{\mathbf{6}}$ & 0.874 \\
$\mathbf{R}_{\mathbf{1 4}}$ & 0.949 & $\mathbf{R}_{\mathbf{2 4}}$ & 0.900 & $\mathbf{R}_{\mathbf{5}}$ & 0.865 \\
$\mathbf{R}_{\mathbf{1}}$ & 0.948 & $\mathbf{R}_{\mathbf{1 6}}$ & 0.898 & $\mathbf{R}_{\mathbf{1 0}}$ & 0.857 \\
$\mathbf{R}_{\mathbf{4}}$ & 0.939 & $\mathbf{R}_{\mathbf{2 6}}$ & 0.897 & $\mathbf{R}_{\mathbf{9}}$ & 0.856 \\
$\mathbf{R}_{\mathbf{1 8}}$ & 0.927 & $\mathbf{R}_{\mathbf{2 1}}$ & 0.885 & $\mathbf{R}_{\mathbf{8}}$ & 0.849 \\
$\mathbf{R}_{\mathbf{2 0}}$ & 0.925 & $\mathbf{R}_{\mathbf{1 5}}$ & 0.885 & $\mathbf{R}_{\mathbf{2 9}}$ & 0.836 \\
\hline
\end{tabular}

The obtained rankings of referees on Table 12 by the help of the proposed integrated approach is different from the ranking obtained with the existing basketball referee evaluation system. Its reason is, in the proposed approach different importance weights are given to the criteria with AHP method and for ranking the referees WASPAS method is used.

\section{Conclusion}

The starting point of this study was that the importance of the criteria of referee evaluation was not determined in the current evaluation system. First of all, in order to eliminate this deficiency, by taking the opinions of the referees, the weights of the evaluation criteria were determined with the help of AHP method and The ranking of the criteria according to their weight is obtained as follows: Standard, Competition and self- 
esteem, Teamwork, Game control, Foul assessment, Physical state, Disciplinary implementation, Overall performance, Mechanical, Violation assessment.

In addition, in the current evaluation system, a ranking is obtained by considering the total number of points received by the referees on the basis of criteria. In this study WASPAS method which is an MCDM method that combines the results of Weighted Sum Model (WSM) and Weighted Product Model (WPM) is proposed for determining the ranking of basketball referees. By this way, more precise and accurate ranking is obtained. This proposed approach guides the decision makers in the basketball referees' performance evaluation process. In future studies criteria can be evaluated by different decision makers in the field. Also, other MCDM can be applied both in determining the weights of the criteria and ranking of the basketball referees. At the end the obtained results can be compared.

\section{Acknowledgement}

This work was supported by the Department of Scientific Research Projects, Pamukkale University.

\section{References}

[1]. Akçakanat, Ö., Eren, H., Aksoy, E. and Ömürbek, V. (2017). "Performance evaluation by ENTROPY and WASPAS methods at banking sector," Süleyman Demirel University The Journal of Faculty of Economics and Administrative Sciences, vol. 22 (2), pp. 285-300.

[2]. Baušys, R. and Juodagalvienè, B. (2017). "Garage location selection for residential house by WASPAS-SVNS method," Journal of Civil Engineering and Management, vol. 23(3), pp. 421429.

[3]. Bingham, A. (1998). Çocuklarda Problem Çözme Yeteneklerinin Geliştirilmesi. (Development of Problem Solving Skills in Children). Milli Eğitim Basımevi, İstanbul.

[4]. Caputo, A. C., Pelagagge, P.M. and Salini, P. (2013). "AHP-based methodology for selecting safety devices of industrial machinery," Safety Science, vol. 53, pp. 202-218.

[5]. Chakraborty, S., Bhattacharyya, O. Zavadskas E. K. and Antucheviciene, J. (2015). "Application of WASPAS method as an optimization tool in non-traditional machining processes," Information Technology and Control, vol. 44(1), pp. 77-88.

[6]. Chakraborty, S. and Zavadskas, E.K. (2014). "Applications of WASPAS method in manufacturing decision making," Informatica, vol. 25(1), pp. 1-20.

[7]. Dağdeviren, M., Yavuz, S. and Kılınç, N. (2009). "Weapon selection using the AHP and TOPSIS methods under fuzzy environment," Expert Systems with Applications, vol. 36, pp. 81438151.

[8]. Heppner, P. P. and Krauskopf C. J. (1987). "The Integration of Personel Problem Solving Processes within Counseling," The Counseling Psychologist. vol. 15, pp. 371-447.

[9]. Karabašević, D., Stanujkić, D., Urošević, S. and Maksimović, M. (2016). "An approach to personnel selection based on SWARA and WASPAS methods," Journal of Economics, Management and Informatics, vol. 7(1), pp. 1-11. 
[10]. Karande, P., Zavadskas E. K. and Chakraborty S. (2016). "A study on the ranking performance of some MCDM methods for industrial robot selection problems," International Journal of Industrial Engineering Computations, vol. 7, pp. 399-422.

[11]. Madić, M., Gecevska, V., Radovanović, M. and Petković, D. (2014). "Multi-criteria economic analysis of machining processes using the WASPAS method," Journal of Production Engineering, vol. 17(2), pp. 79-82.

[12]. Nezhad, M.R.G., Zolfani, S. H., Moztarzadeh, F., Zavadskas, E. K. and Bahrami, M. (2015). "Planning the priority of high tech industries based on SWARA-WASPAS methodology: The case of the nanotechnology industry in Iran," Economic Research-Ekonomska Istraživanja, vol. 28(1), pp. 1111-1137.

[13]. Nie, R., Wang, J. and Zhang H. (2017). "Solving solar-wind power station location problem using an extended weighted aggregated sum product assessment (WASPAS) technique with interval neutrosophic sets," Symmetry, vol. 9(106), pp. 1-20.

[14]. Russo, R.F.S.M. and Camanho R. (2015). "Criteria in AHP: a systematic review of literature," Procedia Computer Science, vol. 55, pp. $1123-1132$.

[15]. Saaty, T. L. (2013), Mathematical Principles of Decision Making: The Complete Theory of the Analytic Hierarchy Process, RWS Publications, USA.

[16]. Saaty, T.L., 1980. "The Analytic Hierarchy Process." McGraw-Hill, New York.

[17]. Stojic, G., Stevic, Ž., Antucheviciene, J., Pamucar, D. and Vasiljevic, M. (2018). "A novel rough WASPAS approach for supplier selection in a company manufacturing PVC carpentry products," Information, vol. 9(121), pp. 1-16.

[18]. Urosevic, S. Karabasevic, D., Stanujkic, D. and Maksimovic, M. (2017). "An approach to personnel selection in the tourism industry based on the SWARA and the WASPAS methods," Economic Computation and Economic Cybernetics Studies and Research, vol. 51(1), pp. 75-88.

[19]. Vaidya, O. S. and Kumar, S. (2006). "Analytic hierarchy process: An overview of applications," European Journal of Operational Research, vol. 169, pp. 1-29.

[20]. Wang, L., Chu, J. and Wu, J., 2007, "Selection of optimum maintenance strategies based on a fuzzy analytic hierarchy process," International Journal of Production Economics, vol. 107, pp. 151-163.

[21]. Yurdoğlu, H. and Kundakcl, N. (2017). "Server selection with SWARA and WASPAS methods," Balıkesir University the Journal of Social Sciences Institute, vol. 20(38), pp. 161173.

[22]. Zavadskas, E.K., Turskis, Z. Antucheviciene, J. and Zakarevicius, A. (2012). “Optimization of weighted aggregated sum product assessment", Electronics and Electrical Engineering, vol.6, pp.3-6.

[23]. Zavadskas, E. K., Saparauskas, J. and Turskis, Z. (2013). "MCDM methods WASPAS and MULTIMOORA: verification of robustness of methods when assessing alternative solutions," Economic Computation and Economic Cybernetics Studies and Research, vol. 47(2), pp. 1-16.

[24]. Zavadskas, E. K., Turskis, Z. and Antucheviciene, J. (2015A). "Selecting a contractor by using a novel method for multiple attribute analysis: weighted aggregated sum product 


\section{$2^{\text {nd }}$ International Conference on BUSINESS, MANAGEMENT \& ECONOMICS}

assessment with grey values (WASPAS-G)," Studies in Informatics and Control, vol. 24(2), pp. 141-150.

[25]. Zavadskas, E. K. Baušys, R. and Lazauskas, M. (2015B). "Sustainable assessment of alternative sites for the construction of a waste incineration plant by applying WASPAS method with single-valued neutrosophic set," Sustainability, vol. 7, pp. 15923-15936.

[26]. Zavadskas, E. K. Baušys, R., Stanujkic, D. and Magdalinovic-Kalinovic M. (2016A). "Selection of lead-zinc flotation circuit design by applying WASPAS method with single-valued neutrosophic set," Acta Montanistica Slovaca, vol. 21(2), pp. 85-92.

[27]. Zavadskas, E. K. Kalibatas, D. and Kalibatie D. (2016B). "A multi-attribute assessment using WASPAS for choosing an optimal indoor environment," Archives of Civil and Mechanical Engineering, vol. 16, pp. 76-85. 